www.jmscr.igmpublication.org

Impact Factor 5.84

Index Copernicus Value: 83.27

ISSN (e)-2347-176x ISSN (p) 2455-0450

crossref DOI: _https://dx.doi.org/10.18535/jmscr/v5i8.11

Journal Of Medical Science And Clinical Research

\title{
Prognostic factors in Spontaneous Hypertensive Intracerebral Hemorrhage
}

\author{
Authors \\ Dr Ranjit Sanu Watson', Dr Aruna ${ }^{2}$, Dr Wilson Joseph ${ }^{3}$, Dr Sabarisree $\mathbf{M}^{4}$ \\ ${ }^{1}$ Associate Professor in General Medicine, Govt Medical College, Kottayam \\ ${ }^{2}$ Additional Professor in General Medicine, Govt Medical College, Trivandrum \\ ${ }^{3}$ Professor in General Medicine, Sree Gokulam Medical College, Trivandrum \\ ${ }^{4}$ Associate Professor (CAP) in Orthopaedics, Govt Medical College, Trivandrum \\ Corresponding Author \\ Dr Sabarisree M \\ Associate Professor (CAP) in Orthopaedics, Govt Medical College, Trivandrum
}

\begin{abstract}
Background: Stroke is a major cause of death and disability. Haemorrhagic strokes constitute $20 \%$ of all strokes. Most important risk factor for development of spontaneous intracerebral haemorrhage is systemic hypertension.
\end{abstract}

Objectives: To study the different clinical and radiologic factors which can be considered as prognostic markers and predictors of survival in patients with spontaneous hypertensive intracerebral hemorrhage.

Materials and Methods: The study is a prospective descriptive study of 100 consecutive patients meeting the inclusion criteria for spontaneous hypertensive intracerebral hemorrhage admitted in the medical wards of a tertiary care centre, Gov. Medical college, Thiruvananthapuram, Kerala. The study was conducted over a period of one year. The patients were clinically assessed and radiologic findings noted.

Results: Older age, presence of headache, vomiting and altered level of consciousness, high systolic BP on admission, long standing hypertension, irregularities in respiration, low GCS on admission, bilateral extensor plantar response, progressive worsening of sensorium, aspiration pneumonia, larger volume of hematoma, presence of intraventricular extension, hydrocephalus, midline shift and mass effect correlated closely with poor outcome and higher mortality.

Conclusions: A low Glasgow coma score on admission, respiratory irregularity, anisocoria, large volume hemorrhage, presence of intraventricular extension and hydrocephalus are statistically significant prognostic markers in patients with spontaneous hypertensive intracerebral hemorrhage.

Keywords: Hypertension, Intracerebral hemorrhage, Stroke.

\section{Introduction}

Stroke is a clinical syndrome characterized by acute development of signs and symptoms of cerebral dysfunction due to a cerebrovascular pathology ${ }^{4}$. Strokes are classified into arterial and venous strokes. Arterial strokes are further classified into ischemic (80\%) and haemorrhagic stroke (20\%). Haemorrhagic strokes are further classified into intracerebral hemorrhage (15\%) and subarachnoid hemorrhage $(5 \%)^{5}$. The most important risk factor for intraparenchymal cerebral hemorrhage is systemic hypertension. Putamen, thalamus, lobes, cerebellum and pons are the most common sites involved in 
intracerebral hemorrhage ${ }^{6}$. The mortality remains high in spite of the developments in surgical and intensive care. So this study aims at finding out the prognostic factors in intracerebral hemorrhage.

\section{Objectives}

To study and analyse the various clinical and radiologic factors which are the prognostic markers and predictors of survival in patients with spontaneous hypertensive intracerebral hemorrhage.

\section{Methods}

This study was conducted as a prospective study on 100 consecutive patients admitted with intracerebral hemorrhage in the medical wards of Govt. Medical College, Thiruvananthapuram, Kerala, a tertiary tier health care facility, over a period of 1 year in 2003-2004. For this study we selected patients aged more than 40 years with a past history of systemic hypertension, with the first clinical development of stroke symptoms and the CT brain showing parenchymal intracerebral hemorrhage. Patients without history of systemic hypertension, patients with past history of stroke, past history of diabetes or any significant chronic ailment, patients on drugs which affect the platelet function or coagulation cascade, patients with head trauma, patients with subdural, epidural or subarachnoid hemorrhage were excluded from the study.

A detailed proforma including the salient points in history, past history, detailed neurological examination findings and radiological findings was prepared. Each patient was seen on the first day of inpatient admission and the clinical evaluation done after getting written informed consent from the care givers. Non-contrast CT scan of brain was taken. The volume of hematoma in millilitres was assessed by the formula A.B.C/2 where $A=$ maximum diameter of the bleed in $\mathrm{cm}$, $\mathrm{B}=$ length of the line perpendicular to the first in $\mathrm{cm}$ and $\mathrm{C}=$ the vertical height of bleed in $\mathrm{cm}$ assessed by the number of axial sections in which bleed is seen ${ }^{1}$ Hydrocephalus was defined as the presence of a dilated contralateral temporal horn of lateral ventricle or a unilateral dilation of frontal and occipital horn of lateral ventricle or presence of bilateral dilated lateral ventricle with or without a dilated third ventricle. ${ }^{2}$

These patients were assessed daily till discharge or death for any neurologic or non-neurologic complications. Patients who developed fever or respiratory symptoms or signs underwent appropriate investigations to rule out infective foci. Follow up was done as outpatient at 4 weeks and 12 weeks.

The data obtained was charted out. P-value $<0.05$ was considered statistically significant, $<0.01$ as very significant and $<0.001$ as very highly significant. Analysis was done using the software SPSS for windows.

\section{Results}

The study was conducted on 100 patients with intracerebral hemorrhage. There were 59 males and 41 females in the study. The male: female ratio was $1.43: 1$. P-value was $>0.05$. So the sex of the patient was not found to be a significant prognostic marker. $7 \%$ of patients were in $40-49$ year age group. $25 \%$ in 50-59 yr age group. 31\%in 60-69 year age group. $27 \%$ in the $70-79$ year age group. $10 \%$ in the $>80$ yrs age group. Overall 3 month mortality in the study was $47 \%$.

47\% patients reported headache as an early symptom in the first $24 \mathrm{hrs}$. The p-value was $<0.001$ which was statistically very highly significant. So, the conclusion was that, patients who reported headache as an early symptom was having a significantly higher mortality. In our study, cerebellar bleed had $71.4 \%$ chance of headache while thalamic bleeds had $34.3 \%$ chance of development of headache. Seizures were seen only in $15 \%$ of the patients. All except one were partial seizures with secondary generalization. The Chi-square value was 2.74 , the Odds ratio was 2.595 , p-value $>0.05$. so presence of seizures increased the mortality rate but was not statistically significant to be of prognostic significance. Vomiting was reported by $72 \%$ 
patients. The $\mathrm{p}$-value $<0.01$. So vomiting is a significant prognostic factor. Almost equal numbers of patients were present in the study with right and left hemiplegia. 44\% patients with right hemiparesis/plegia expired, while $41 \%$ with left hemiparesis/plegia expired. This difference was not statistically significant, $\mathrm{p}>0.05$.

The duration of hypertension was analysed to know whether it had any correlation with the prognosis. The Chi-square value was 7.967 . The p-value $<0.05$, which was statistically significant. So, the longer duration of hypertension was associated with a poor prognosis. Only $32 \%$ of the patients were on regular medical check-ups before the stroke. But all the patients enrolled in the study were aware that they had hypertension. So poor drug compliance and longer duration of hypertension can be considered as poor prognostic markers. Statistical analysis to find out whether smoking was of any prognostic significance was done. The risk estimate by Odds ratio for smoking was 0.852 . The Pearson Chi-square value was 0.147 . The $p$-value was $>0.05$. So smoking was not found as a significant prognostic marker. There were 24 alcoholics in the study. Odds ratio for alcoholism was 0.600 , Chi-square value was 1.144 , the p-value $>0.05$. So, alcoholism was not a significant prognostic marker. The systolic blood pressure on admission was assessed. The Chisquare value was 10.242 , p-value $<0.05$, was statistically significant. So, higher the systolic blood pressures on admission poorer the prognosis.

Respiratory pattern on admission and the immediate post admission period of one week was assessed. The Pearsons Chi-Square value was 27.915, p-value $<0.001$. So, irregular ataxic Biot's breathing or Cheyne-Stoke's breathing carries a poor prognostic value. Glasgow Coma score on admission was assessed. The Chi-square value was 49.987 , the p-value was $<0.001$. So the GCS on admission was statistically very significant and therefore a very significant marker of prognosis. Bilateral extensor plantar response was also found to be highly significant a prognostic marker with p-value <0.001. Chi-square value 30.268. Anisocoria was found to be a marker of poor prognosis in IC bleed, with p- value $<0.001$, Chi square value 12.53 . All these patients had a very poor survival rate and the mortality rate was $100 \%$ for patients with anisocoria in our study. The voltage criteria in ECG was used as an evidence of left ventricular hypertrophy. The chi-square value was 0.525 and the $p$-value was $>0.05$. So the presence of $\mathrm{LVH}$ does not seem to be of significance in the 3 month survival rates of patients with intracerebral hemorrhage.

The importance of site of bleed in the prognosis of intracerebral hemorrhage was assessed. The Pearsons chi-square test value was7.878 and pvalue $<0.05$. So, the site of bleed was found to be a significant predictor of prognosis. Mortality was maximum $(85.7 \%)$ in pontine hemorrhage, least for basal ganglia hemorrhage $(37.3 \%)$. The volume of hematoma was assessed from the images. It was found to be a highly significant marker in prognosis of intra cerebral bleed, with p-value $<0.01$ and Chi-square value 19.020. The larger the hematoma, poorer the outcome. The presence of intra ventricular extension was assessed from the CT images. $43 \%$ of patients had intra ventricular extension of hemorrhage. The $\mathrm{p}$ value was $<0.001$ and the odds ratio value was 10.136, chi- square value was 26.793. So the presence of intraventricular extension is therefore a very poor prognostic sign. Similarly, presence of hydrocephalus in CT images was also found to be a highly significant poor prognostic marker in intracerebral hemorrhage. (p-value <0.001, Odds ratio 15.167, Pearson Chi-square value 27.071). In our study it was also observed that, $96 \%$ had intra ventricular extension of hemorrhage.

Progressive worsening of sensorium after admission was also found to be a highly significant poor prognostic marker (p-value $<0.001$, Odds ratio 12.468, chi-square value 28.876). Complications like pneumonia (Chi square value 5.606 , $\mathrm{p}$ - value $<0.05$, odds ratio 3.671) was found to be a statistically significant poor prognostic factor while urinary tract 
infection, bedsores and SIADH were not found so.

\section{Discussion}

The patients who were studied were diagnosed much earlier as having systemic hypertension and of age >40 years, presently admitted with CT proven intra cerebral hemorrhage. The patients who developed hemorrhage in the classical sitesfor hypertensive intra cerebral hemorrhage were included in the study, which included basal ganglia, thalamus, cerebellum and pons ${ }^{7}$ Lobar hemorrhage was excluded from the study because cerebral amyloid angiopathy would also be a significant contributor to lobar bleed and could be a major confounding variable. There were 59 males and 41 females in our study. The sex ratio was $1.43: 1$. This ratio was comparable to the 1.38:1 ratio obtained in the Reese Hospital registry for strokes ${ }^{7}$. The patients were classified according to the age. Maximum incidence was noted in the 60-69 year age group. Essential hypertension usually starts at around 40 years ${ }^{3}$ and end organ damage may occur by around 20 years which may have led to the peak incidence in this age group. In the Greater Cincinnati study ${ }^{8}$ the maximum incidence was in the age 70-79 years. They had included all patients with spontaneous intra cerebral hemorrhage. Hypertensive bleeds were maximum in the 60-69 year age group. In this age group of 60-69 the males outnumbered females in the ratio 2.09:1. In our study, in the age group of 60-69, the sex ratio was 2.1:1. The final end points taken were death or survival. The mortality was $47 \%$ in our study. The various studies in the pre CT and post CT era show wide ranges in mortality ${ }^{4}$. Those differences could be due to the fact that, different authors used different time periods for assessing the mortality. The rates were as low as $20 \%$ in the study by Bogousslavsky et al ${ }^{9}$ to as high as $56 \%$ in the study by silver et $\mathrm{al}^{10 .}$. The 30 day mortality is $47 \%$ in our study and correlates well with other studies. Though intra cerebral bleeds are considered to occur during exertion in our study it was found that $67 \%$ of subjects were at rest or in minimal exertion at the onset of symptoms. Only 33\% reported that the symptoms started while on exertion. There was no prognostic relation noted between those patients who developed symptoms on exertion and those who did not. In the data from the Michael Reese Stroke Registry 13\% had intra cerebral bleed on waking up from sleep, $10 \%$ during moderate to maximum exercise , 64\% during minimal activities of daily living and $10 \%$ at unknown levels of exertion ${ }^{7}$. Headache was a symptom reported by $47 \%$ of our patients, but vomiting was seen in $72 \%$. The lower incidence of headache may be due to the fact that, vomiting is often an objective finding which is easily perceived by the care givers but headache may not be complained by those patients who may suddenly lapse into unconsciousness. Headache as a symptom was seen in $41 \%$ of the 237 patients in the Stroke Data Bank ${ }^{12}$ and $40 \%$ in the Lausanne Stroke Registry. ${ }^{11}$. Headache and vomiting is often associated with larger bleeds and in the presence of intra ventricular extension. So both headache and vomiting early in the disease course are considered as significant markers of poor prognosis. In our study $15 \%$ of patients had seizures. The values were comparable to the $14 \%$ incidence reported in the study by Kilpatrick et $\mathrm{al}^{13}$. Seizures as a prognostic marker were statistically not significant. The duration between the onset of symptoms and the time taken to reach the hospital was assessed. Studies on acute myocardial infarction have conclusively shown the importance of index pain and window period in the final outcome of patients. Whether similar relationship exists for intra cerebral hemorrhage was considered. Prognostic significance in the form of $\mathrm{p}$-value $<0.05$ was obtained and this fact needs to be confirmed by larger studies. In the analysis of this variable, fallacies can occur because the patients may be brought to the hospital in less severe bleeds and may be brought earlier to the tertiary care centre in more severe bleed. 
In this study, those patients with $>10$ years history of hypertension had $67 \%$ mortality compared to $35 \%$ in those with duration of hypertension $<5$ years. No significant relation to mortality or poor prognosis was noted in smokers and alcoholics. High systolic blood pressure is a risk factor responsible for progressive enlargement of the hematoma and cerebral oedema. Moreover larger bleeds tend to activate the Cushing's reflex leading to further rise in systolic blood pressure. Irregular respiration is a bad prognostic factor as it often implies direct or indirect involvement of the pontine or medullary respiratory centres. Even supratentorial bleeds, if sufficiently large can compress, shift or produce torsion of brain stem structures compensating the respiratory centres ${ }^{14}$. In our study, $56 \%$ of patients of patients had a Glasgow Coma Score of $<10$ on admission. GCS on admission is a very strong predictor of the prognosis in patients with intracerebral hemorrhage. Higher values are associated with a better prognosis. Deep coma on presentation carries a high mortality according to the studies by Portenoy et $\mathrm{al}^{15}$. The most reliable and consistent clinical predictor of outcome in intracerebral hemorrhage is the level of consciousness as the patient is wheeled into the emergency department. In our study, the mortality rate for patients with GCS <6 was $92 \%$. Similarly, progressive worsening of sensorium, bilateral extensor plantar and anisocoria predict a grave prognosis. The worsening of sensorium is attributed often to the enlargement of bleed or associated oedema or development of hydrocephalus which are all independent predictors of a poor prognosis. Anisocoria is secondary to medial uncal herniation and its pressure on the third cranial nerve which courses medial to the uncus.

CT brain was also assessed for the poor prognostic factors. Site of bleed, volume of the hemorrhage, presence of intra ventricular extension, presence of hydrocephalus and mass effect were independent predictors of a poorer prognosis. Pontine and cerebellar bleed had the highest mortality of $85 \%$ and $71 \%$ respectively. The reasons may be that these bleeds are into the restricted posterior cranial fossa where even a small increase in brain volume may precipitate a herniation of cerebellar tonsilar herniation and death. In our study, intra ventricular extension was seen in $37.3 \%$. Thalamic hematomas carry higher incidence of intra ventricular extension (60\%), most probably because thalamus forms the lateral boundary of the third ventricle and therefore bleeds tend to track down into the third ventricle. The blood in the ventricles leads to clogging of the CSF pathways leading to obstructive hydrocephalus and further worsening of sensorium. The study by Stein et al16.

The mean hematoma volume was $15.6 \mathrm{ml}$. hematoma volumes $>40 \mathrm{ml}$ is found to have significantly higher mortality. The hematoma size on admission CT has shown a high predictive value for short term mortality. The correlation is more with supratentorial hematomas. Hematoma volume $>60-80 \mathrm{ml}$ had $100 \%$ mortality despite the site.

\section{Conclusion}

Older age, presence of headache, vomiting and altered level of consciousness, high systolic BP on admission, long standing hypertension, irregularities in respiration, low GCS on admission, bilateral extensor plantar response, progressive worsening of sensorium, aspiration pneumonia, large volume bleed, bleed with intra ventricular extension, hydrocephalus, midline shift and mass effect correlated closely with poor prognosis and higher mortality in patients with hypertensive intracerebral hemorrhage.

\section{Bibliography}

1. The ABCs of measuring intracerebral hemorrhage volumes- Rashmi U. Kothari. Thomas Brott, Joseph P. Broderick, William G.Barsan, Laura R Sauerbeck, Mario Zuccarello, Jane Khoury. Stroke 1996; 27:1304-1305

2. The definition and classification of hydrocephalus: a personal recommendda- 
tion to stimulate debate: Harold L Lekate Cerebrospinal Fluid Research2008 5:2

3. Intensive Blood-Pressure Lowering in Patients with Acute Cerebral Hemorrhage Adnan I. Qureshi, M.D., Yuko Y. Palesch, Ph.D., William G. Barsan, M.D., Daniel F. Hanley, M.D., for the ATACH-2 Trial Investigators and the Neurological Emergency Treatment Trials Network: N Engl J Med 2016; 375:1033-1043

4. Acute Ischemic Stroke H. Bart van der Worp, M.D., Ph.D., and Jan van Gijn, F.R.C.P.NewEngl J Med 2007; 357:572579August 9, 2007

5. Panagariya. A, AK sharma, V Sardana, A. maru An analysis of 141 cases of intracerebral hemorrhage: A study from north India: Neurosciences today 2000 4:196-199

6. Long-Term Prognosis of Hypertensive Intracerebral Hemorrhage Michael A Douglas MD and Armin F Haerer Stroke, Vol 13, No 4, 1982

7. Caplan LR, Hier DB, D'cruz I, Michael Reese Stroke Stroke Registry: Stroke 1992; 14:530-536

8. The Greater Cincinnati/Northern Kentucky Stroke Study: preliminary first-ever and total incidence rates of stroke. Broderick $\mathbf{J}^{1}$, Brott T, Kothari R, Miller R, Khoury J, Pancioli A, Gebel J, Mills D, Minneci L, Shukla R.

9. Bougousslavsky J, Dartigues JF et al Death and functional outcome after spontaneous intracerebral hemorrhage: a prospective study of 166 cases using multivariate analysis Stroke: 1995; 22:1-6

10. Silver FL, Norris JW, Lewis AJ et al. early mortality following stroke: a prospective review. Stroke 1988;15:492-496

11. Van Melle, bogouslavsky J, Regli.F The Lausanne Stroke Registry: analysis of
1000 patients with the first stroke. Stroke 1997;19:1083-1092

12. Foulkes MA, Wolf PA, Price TR, Mohr JP, Hier DB. The Stroke Data Bank: design, methods and baseline characteristics. Stroke 1988; 19:547-554

13. Kilpatrick CJ, Davis SM, Tress BM et al. Epileptic Seizures in acute stroke, Arch Neurology 1990;47:157-160

14. Mc Cormick, rosenfield J; Respiratory pattern is a determinant of early mortality in putaminal hemorrhage.

15. Portenoy RK, Lipton RB, Berger AR et al. Intracerebral hemorrhage: a model for the prediction of outcome $\mathbf{J}$ Neurology Neurosurgery Psychiatry 1992;50:976-979

16. Stein RW, Caplan LR et al: Outcome of intracranial hemorrhage. Role of blood pressure and location and size of the lesions. Annals of Neurology 1993; 14:132-133. 